\title{
L'art, la littérature et le vivant
}

De l'épistémique à l'esthétique... et retour!

\section{Laurence Dahan-Gaida}

\section{(2) OpenEdition}

Journals

Édition électronique

URL : http://journals.openedition.org/aes/894

DOI : $10.4000 /$ aes.894

ISSN : 2258-093X

Éditeur

Laboratoire LISAA

Référence électronique

Laurence Dahan-Gaida, «L'art, la littérature et le vivant », Arts et Savoirs [En ligne], 7 | 2016, mis en ligne

le 13 décembre 2016, consulté le 30 avril 2019. URL : http://journals.openedition.org/aes/894 ; DOI :

$10.4000 /$ aes. 894

Ce document a été généré automatiquement le 30 avril 2019.

Centre de recherche LISAA (Littératures SAvoirs et Arts) 


\title{
L'art, la littérature et le vivant
}

\author{
De l'épistémique à l'esthétique... et retour!
}

\author{
Laurence Dahan-Gaida
}

\section{Introduction}

1 Très tôt, les formes du vivant ont été mises en rapport avec les formes de l'art par des penseurs et des créateurs venus des horizons les plus divers. L'analogie entre les lois du vivant et celles de la création artistique a emprunté différentes voies, à commencer par celle de la forme, dont la genèse " entre le vide et l'événement pur " ${ }^{1}$, a fasciné poètes, artistes, philosophes et historiens de l'art.

2 Mais la forme n'existe pas seulement dans sa genèse : pour s'accomplir véritablement, elle doit encore être perçue, devenant alors affaire de vision et de cognition. Ici se noue un second lien entre les sciences du vivant et l'esthétique, unies dans la même quête d'un cadre théorique capable d'unifier les processus perceptifs et l'esthétique.

Or l'esthétique n'est pas seulement une science des formes et de leur réception, c'est aussi une science du beau qui a sans cesse mis en rapport les «belles formes» de l'art avec la beauté du vivant, érigé tour à tour en modèle à imiter ou en idéal de perfection inaccessible. Et c'est ici que se noue le troisième nœud entre l'art et le vivant.

Dans les pages qui suivent, je me propose d'explorer quelques-unes de ces modalités d'échange entre les deux domaines, sans toutefois prétendre à l'exhausitivité ou à la continuité historique. Je ne me préoccuperai fondamentalement ni de genèse ni de causalité mais je tenterai plutôt de montrer comment l'imagination et la pensée se retrouvent lorsqu'il s'agit de rapprocher la création naturelle de la création artistique.

\section{«Les coraux de Darwin » : quand la science devient art...}

Dans Les coraux de Darwin, Horst Bredekamp a montré l'importance que le naturaliste anglais accordait à l'esthétique dans sa formalisation de la lutte pour la survie. Darwin 
était en effet convaincu que la grande bataille de la sélection naturelle avait pour contrepartie un principe esthétique grâce auquel les forces impliquées dans la lutte pour la vie pouvaient s'épanouir dans la beauté des productions naturelles. Or ce principe, il l'a trouvé dans le corail, image d'une force vitale qui témoignait à ses yeux de la variabilité de la nature et de sa créativité anarchique, contredisant en cela toutes les images d'ordre véhiculées par le modèle de l'arbre ${ }^{2}$. Si Darwin a longtemps hésité entre l'image d'un arbre plus ou moins généalogique et celle d'un buissonnement corallien pour représenter l'évolution, c'est que les deux images recouvrent des choix intellectuels différents. Penser " en arbre " n'est pas la même chose que penser «en tableau » ou penser «en carte ", chacun de ces modèles graphiques mettant en œuvre un schème mental particulier, une rationalité spécifique. Alors que l'image de l'arbre souligne les différences et les écarts entre les espèces, le schème du corail tire sa force de conviction de ce que ses branches et ses ramifications peuvent toujours produire de nouvelles unions, même après leur division. Le fait que les coraux, dans leur croissance, ne tendent pas vers un but défini, constitue la réfutation d'une téléologie des fittest marquée par l'étroitesse du darwinisme social, en même temps que de la certitude créationniste d'un plan fondateur ${ }^{3}$. Mais si le corail permet de visualiser l'action du temps de façon si significative, c'est aussi parce qu'il rend visible d'un seul coup d'œil la séparation entre les espèces encore vivantes et les espèces éteintes. Filant la métaphore du fossile, Darwin montre comment les espèces vivantes actuelles ont émergé d'espèces disparues, figurées par les troncs et les bras pétrifiés du corail : "L'arbre de vie devrait peut-être être nommé le corail de la vie", écrit-il dans ses Notebooks 4 . Si Darwin préfère l'image du corail à celle de l'arbre, c'est que ses troncs morts peuvent être interprétés comme des fossiles des espèces mortes et que ses branches s'écartant l'une de l'autre offrent un modèle alternatif à la thèse lamarckienne de la transformation continuelle : «Base des branches mortes; de sorte que les passages ne peuvent être vus - cela contredit à nouveau la succession constante des germes en progression. $»^{5}$

6 Mais c'est aussi par sa dimension esthétique que le corail fascine Darwin : voyant en lui le symbole d'une pacification de la mer et de la terre, il célèbre avec une verve toute littéraire les édifices longs de plusieurs kilomètres que les coraux construisent pendant des millénaires, par une infinité de processus infimes dont la puissance surpasse toute force mécanique ${ }^{6}$. Darwin souligne l'activité infatigable du corail qui lui permet, lentement mais sûrement, de construire des édifices immenses, plus impressionnants que les plus grandes ruines de l'Antiquité7. Pour Darwin comme pour ses contemporains, le corail participe d'une esthétique enchanteresse qui s'inscrit dans une tradition maintenue jusqu'au $\mathrm{xIX}^{\mathrm{e}}$ siècle, qui faisait du corail l'emblème de toutes les métamorphoses. Déjà dans l'Antiquité, Ovide avait décrit les coraux comme des « artistes de la transformation $»^{8}$ ainsi qu'en témoignent ces vers souvent cités : "Il en résulte aujourd'hui le corail, qui a même propriété, / Durcissant au contact de l'air : ainsi, ce qui dans l'eau était/Plante souple devient hors de l'eau une pierre $»^{9}$. Objet naturel, qui peut être travaillé par l'homme et ainsi transformé en œuvre d'art, le corail a suscité un engouement sans précédent à l'époque de Darwin, fascination qui fut sans doute accentuée par la passion de ses contemporains pour les aquariums.

7 Tout en soulignant la puissance esthétique d'un objet naturel comme le corail, Darwin l'a transformé en dispositif épistémologique, créateur de sens. C'est la même démarche qui s'exprime dans son principe de "sélection sexuelle», complément de la "sélection naturelle ", qui fait apparaître l'évolution non plus comme une force aveugle mais comme 
un processus orienté par un principe esthétique ${ }^{10}$. La biologie n'a reconnu que récemment la possible pertinence de cette théorie qui fait appel au principe de la beauté pour expliquer, au- delà de la lutte pour la survie, le choix de leur partenaire par les individus : guidés par des critères esthétiques, ces derniers choisiraient des partenaires susceptibles d'assurer la perpétuation, de génération en génération, de qualités mentales et physiques impliquées dans la séduction, dont Darwin pensait qu'elle jouait un rôle essentiel dans l'évolution. Le privilège accordé par Darwin à l'œil des femelles a conduit certains spécialistes d'esthétique, de littérature et d'histoire de l'art à souligner le rôle de cet organe comme agent de l'évolution, érigeant ainsi l'émotion esthétique au rang de principale pulsion de la sélection sexuelle et, partant, de la transformation des espèces ${ }^{11}$.

\section{La morphologie de Goethe}

8 Si Darwin mobilise l'esthétique avant tout comme science du beau, c'est à Goethe qu'il revient d'avoir, le premier, élaboré une théorie de la forme capable de rendre compte des lois du vivant aussi bien que des lois de l'art. Au XIX ${ }^{e}$ siècle, les théories goethéennes sur l'émergence des formes naturelles ont intéressé les scientifiques aussi bien que les écrivains, contribuant à tisser des liens entre dynamique de la forme et discours de la biologie qui se maintiennent aujourd'hui encore ${ }^{12}$. La pratique de Goethe est d'abord une pratique des sciences du vivant, une pratique de l'anatomie et de la botanique, puis de la physiologie, sciences qu'il cherche à articuler en un tout cohérent qu'il appelle significativement "morphologie», par quoi il entend la "théorie de la forme, de la formation et de la transformation des corps organiques $»^{13}$. En tant que telle, la morphologie trouve sa place à l'intérieur de l'histoire naturelle comme étude des formes des parties de la nature organisées en relation avec leur tout vivant. Or le vivant se définit moins par sa forme que par sa formation: il est conformation et connexion, c'est-à-dire interaction avec le milieu de vie. Goethe distingue en effet la forme comme structure, la forme phénoménale fixe (Gestalt) de la forme comme construction ou comme activité de conformation (Bildung). Ce processus formateur, il le saisit à travers le concept scientifique et philosophique de métamorphose qui permet de comprendre la manière dont les lois de la nature s'investissent dans la concrétude empirique. Dès lors, la connaissance des formes passera par l'appréhension de leurs mouvements, changements, développements dans le temps de la nature. Ce qui implique un point de vue dynamique, fondé sur l'interaction du sujet et de l'objet dans le processus de connaissance, justifiant l'usage du terme de Bildung de préférence à celui de Gestalt :

L'allemand désigne l'ensemble du donné (Komplex des Daseins) propre à un être réel par le terme de Gestalt (forme). En usant de cette expression, il fait abstraction de ce qui est en mouvement et assume qu'un ensemble cohérent est arrêté, délimité et fixé par son caractère. Si nous considérons cependant l'ensemble des formes et surtout des formes organiques, nous constatons que nulle part ce qui est stable, en repos et délimité ne prédomine, mais qu'au contraire tout est pris dans un mouvement constant. De là vient que notre langue use avec assez d'à-propos du terme de Bildung (formation) pour désigner aussi bien ce qui est produit que ce qui est en train de l'être. Si nous voulons donc introduire à une morphologie, nous ne parlerons pas de conformation, et si nous employons ce terme, nous ne penserons tout au plus qu'à l'idée, au concept, ou à un élément fixé pour un instant seulement par l'expérience connaissante. ${ }^{14}$

9 Dans la morphologie goethéenne, les termes de Bildung et de Gestalt ne se séparent pas de ceux de force formatrice (Bildende Kraft), de pulsion (Trieb) et de structure au sens des 
relations entre Tout et Parties (relations méréologiques) ${ }^{15}$. C'est sur ce dernier point que les systèmes de l'art et de la nature vont converger : dans l'idée d'un tout qui lie les parties, d'une totalité organique capable de traduire la cohérence souterraine de la vie derrière l'inépuisable diversité des formes qu'elle sécrète. Goethe considère en effet que la forme artistique dérive de la forme vivante, qu'elle en reproduit les caractéristiques essentielles et qu'elle peut donc, en retour, en présenter un modèle d'intelligibilité opératoire, du moins sur le plan heuristique. La forme devient ainsi la clé de compréhension de toutes choses, parce qu'elle permet de jeter un pont entre le monde de l'art et le monde naturel qui, tous deux, résultent d'une productivité spontanée de la nature organisée. C'est en ce sens qu'il faut comprendre les déclarations de Goethe qui investissent l'esthétique d'une valeur paradigmatique pour la morphologie : «Celui à qui la nature commence à dévoiler son secret manifeste, ressent un irrésistible désir pour son interprète le plus digne : l'art " ${ }^{16}$. Dès lors, les sciences de la nature et l'esthétique peuvent avancer de concert puisque « le procès de la nature comme celui des arts est un façonnement, une construction de la forme qui, par le biais de processus cognitifs, peut s'ouvrir sur la sphère du sens $»^{17}$.

Constituant une méthode en soi, la morphologie goethéenne tire son originalité de sa tentative de concilier un regard empirique portant sur la dimension visible de l'organisme et un regard spéculatif portant sur sa dimension invisible, idéelle, soustraite à l'observation. Pour Goethe en effet, la dynamique de génération n'est pas en deçà du sensible, elle a une visibilité de droit car les lois et règles de la forme se donnent dans l'apparaître même (Erscheinung), "sans préconception d'objet " ${ }^{18}$. Dès lors, la forme devient le moyen de dégager « une rationalité à la surface même des phénomènes étudiés, sans descendre sous eux, sans les dissoudre dans autre chose qu'eux-mêmes ${ }^{19}$. Le parti pris épistémologique qui se cache derrière cette démarche est de ne jamais expliquer un phénomène (par exemple l'organe d'une plante) par des éléments explicatifs d'une autre nature que lui-même (par exemple des processus physico-chimiques). La formation d'une étamine, par exemple, ne s'expliquera pas par des processus chimiques sous-jacents mais par les transformations du pétale : "Ainsi une étamine est produite dès que les organes, que jusqu'alors nous avons vus se dilater pour former des pétales, se contractent à un haut degré et paraissent en même temps dans un état beaucoup plus perfectionné $»^{20}$.

11 Dès lors, on comprend mieux pourquoi Goethe refusait la mesure, les mathématiques, l'emploi du nombre et du quantitatif dans l'expérience. Négligeant l'explication mathématique, il ne l'exploitait que pour vérifier la description sensible, souvent esthétisante, qui était censée donner à voir l'essence des choses. Or l'essence goethéenne n'est pas une structure, elle est un pouvoir de structuration ou plutôt de formation, qui est décelable dans la forme visible et qui relève à la fois de l'universel et du singulier. Dans le monde visible, Goethe cherche à repérer une forme caractéristique, sorte d'archétype qui permet d'accéder à la fois aux particularités d'un être unique et au type générique présent, de façon immanente et en puissance, dans la manifestation phénoménale. C'est l' Urpflanze, ou plus généralement l'Urphänomen, dont Goethe conçoit l'idée en 1787, dans le jardin botanique de Palerme, lors de son voyage en Italie de 1786-1788. Par ce terme, il entend une " information primordiale », une sorte de " code génétique » où serait encodé un «style d'information et d'organisation » de la forme ${ }^{21}$. La fonction de l'Urpflanze est de rendre visible ce qui fait d'une plante une plante, la nécessité de son organisation interne mais aussi ce qui la distingue des autres plantes. C'est un principe générateur, un modèle de construction comme l'explique Goethe dans une lettre à Herder du 17 mai 1787 : 
L'Urpflanze sera la créature la plus étrange du monde, et la nature elle-même me l'enviera. Avec ce modèle et la clé qui l'explique, on peut inventer d'autres plantes à l'infini, qui seraient forcément cohérentes, c'est-à-dire qui, même si elles n'existaient pas, pourraient cependant exister, qui ne sont pas des ombres ou des apparences picturales ou poétiques, mais ont une vérité et une nécessité internes.

La même loi pourra s'appliquer à toutes les autres créatures vivantes. ${ }^{22}$

L'Urpflanze doit être comprise comme un modèle idéel et non comme une plante qui serait première chronologiquement et dont toutes les autres seraient issues. Pour autant, elle n'est pas un simple archétype abstrait dont l'opérativité serait seulement logique. Plutôt qu'un schème ou un type générique, c'est un principe générateur d'une variabilité infinie, qui ne prédétermine pas les modalités d'existence de chaque être vivant mais seulement «sa capacité de perfectionnement (progressus perfectionis) » ${ }^{23}$. Didier Hurson parle à cet égard d'une «pensée du vivant comme pensée d'un ordre germinatif et d'une puissance expansive ", s'articulant moins à l'idée de possible - qui suppose un réel en attente - qu'à celle de virtualité24. Face à un phénomène donné, Goethe cherche en effet à retrouver la trajectoire qui va du virtuel à l'actuel: dans les arts plastiques, ce sera par exemple le trajet qui va de la forme imaginée à la forme réalisée; pour les sciences de la nature, il s'agira de saisir l'essence telle qu'elle se manifeste à la perception, dans le pur apparaitre des formes.

Située au croisement créateur de l'essence et de l'événement, la pensée goethéenne de la forme manifeste la liberté de la Nature d'actualiser à l'infini de nouvelles configurations latentes, par modification, complexification, enrichissement de l'essence - de l' Urphänomen - et par relance des possibles ${ }^{25}$. La rationalité morphologique qu'il dessine ainsi s'ancre dans un refus du réductionnisme qui va de pair avec le privilège accordé à la transformation: est «forme " ce qui peut être pensé comme la transformation d'une chose en une autre - c'est-à-dire «ce qui peut être défini dans un ensemble de transformations $»^{26}$-, sans jamais que cette chose se dissolve en ses composantes.

\section{La « morphologie générale » de Paul Valéry}

14 L'idée d'une créativité morphologique commune aux arts et à la nature est aussi l'une des idées directrices de Paul Valéry, qui pressentait l'existence d'une même générativité à l'œuvre dans les variations morphogénétiques de la nature et dans les images créées par l'homme. Valéry s'est intéressé aux forces de "formation systématique des formes », aux transformations et modulations de la nature vivante, qui vont devenir le modèle de ce que Merleau-Ponty appellera plus tard «une morphologie dynamique $»^{27}$. L'étude du vivant doit en effet permettre de considérer « la formation systématique des formes, la recherche de transformations, modulations, etc.» qui œuvrent comme un pouvoir de structuration ${ }^{28}$. Comme Goethe, c'est à l'activité de conformation que s'intéresse Valéry : à la Bildung plutôt qu'à la Gestalt, à la puissance productrice de formes plutôt qu'au produit fini, autrement dit à la dynamique de génération telle qu'elle s'incarne par excellence dans la Nature : «Nature, c'est-à-dire la Produisante ou la Productrice. C'est à elle que nous donnons à produire tout ce que nous ne savons pas faire et qui, pourtant, nous semble fait. $\|^{29}$

La théorie valéryenne est fondée sur cette intuition profonde que la Nature est une force de production et que si l'art doit l'imiter, c'est dans son activité de création de formes : elle doit se réapproprier les « forces formatives » à l'œuvre dans la Nature en confiant au 
logos la charge de les investir. Avant l'homme en effet, la Nature dispose d'une force informante qui, à tous les étages de son organisation, pluralise sans fin le réseau de ses figures possibles. Charge à l'art de se réapproprier cette force afin de produire à son tour des figures d'une égale perfection et variété.

Or pour Valéry, la forme par excellence, c'est la coquille, objet naturel auquel il va consacrer en 1937 un essai devenu célèbre : «L'homme et la coquille». Ce qui fascine Valéry dans la coquille, c'est qu'elle manifeste la perfection des forces-formes de la phusis dont elle fait voir le travail. La coquille, en effet, n'est rien d'autre que la forme rendue visible de forces marines, dont le mouvement en spirale se retrouve dans d'autres tourbillons - oreilles, boucles, ondes, chevelures, etc. Dans son essai de 1937, Valéry insiste sur la résistance épistémologique que la coquille oppose à toute appréhension rationnelle. En effet, contrairement aux objets fabriqués par l'homme, le mystère de la coquille ne se laisse réduire par aucun des moyens de notre connaissance: ni la géométrie, ni la physique, ni la biologie ne parviennent à expliquer le processus générateur qui est à son origine, la dynamique de son engendrement qui est celle du mouvement tourbillonnaire. Le problème posé par la coquille est celui de la finalité de l'organisation: alors que le faire humain obéit à une intention que l'on peut déchiffrer, "la fabrication de la coquille est chose vécue et non faite ${ }^{30}$. Ce qui fait la différence entre production humaine et production naturelle, c'est l'«inhérence organique » de la coquille qui semble littéralement émaner du mollusque, alors que «nos desseins réfléchis et nos constructions ou fabrications voulues semblent très étrangers à notre activité organique profonde $»^{31}$. Autrement dit, le "faire» du mollusque a ceci de particulier qu'il coïncide avec son être, alors que les deux activités semblent disjointes chez l'homme : la coquille produit son identité en produisant sa propre forme, dans une adhérence parfaite entre la production morphologique et l'ontologie.

Valéry semble avoir ainsi anticipé la notion d'autopoïèse, qui a été popularisée par les biologistes chiliens Francisco Varela et Humberto Maturana au début des années 1990 : "Notre proposition est que les êtres vivants sont caractérisés par le fait que, littéralement, ils sont continuellement en train de s'auto-produire. Nous nous référons à ce processus lorsque nous appelons l'organisation qui les définit l'organisation autopoïétique. $\aleph^{32}$ Ce qui fait la singularité des systèmes autopoïétiques, et donc des organismes vivants, c'est une parfaite congruence entre la production morphologique et l'ontologie, et donc entre l'être (l'identité) et le faire (la production de son organisation). Or c'est précisément ce qu'illustre de manière exemplaire le mollusque de Valéry, «être qui ne sait que sa leçon, avec laquelle son existence même se confond $»^{33}$. L'idée d'autopoìèse semble avoir été anticipée par Valéry dès 1920, lorsqu'il écrivait: "L'organisation, la chose organisée, le produit de cette organisation et l'organisant sont inséparables $»^{34}$. En 1937, l'année même où il rédige son essai sur la coquille, il revient sur cette idée, non plus pour définir le monde biologique mais pour décrire l'activité poétique. Dans son cours inaugural de poétique au Collège de France, il avoue en effet avoir un moment songé, pour définir la poétique, à employer le mot de " poïétique » dont «la physiologie se sert quand elle parle des fonctions hématopoïétiques ou galactopoïétiques. $»^{35}$ C'est donc en référence à la biologie que Valéry définit la poétique, ramenant le poïen à une théorie du produire naturel, à un processus de fabrication, voire d'auto-fabrication, qui est à l'œuvre dans le monde biologique. La po(i)étique devient ainsi une sorte de seconde nature, qui manifeste les mêmes lois de génération et de croissance que les organismes vivants et qui peut donc être investie par le logos pour 
produire des formes d'égale beauté que celles produites par la nature. Ce qui avait été séparé dans son texte sur la coquille, le faire naturel et le faire poétique, se trouve ainsi relié par une autre sorte de continuité. Cette continuité est également postulée par des auteurs contemporains, comme par exemple l'écrivain allemand Botho Strauss, qui se réfère explicitement aux thèses de Varela et Maturana pour décrire le système littéraire comme un processus d'auto-génération de l'écrit par l'écrit lui-même :

Comme l'enseigne aujourd'hui une biologie cybernétique, seul ce qui fait référence à soi-même, peut maîtriser la complexité de son environnement. [...] L'auteur réagit moins au monde que, bien plus, à sa propre compréhension du monde; et celle-ci est née avant tout de la littérature. Il est en premier et en dernier ressort un événement marginal dans un livre rempli depuis longtemps. Son œuvre accompagne un moment cette perpétuelle écriture, de laquelle il est venu et à laquelle il retournera à nouveau. ${ }^{36}$

La littérature naît de la littérature, c'est un processus autopoḯtique qui ne fait que transiter par l'auteur et le lecteur - dès lors réduits à de simples épiphénomènes - pour se produire elle-même, selon un mouvement largement autonome. Cette représentation s'inspire du fait que les systèmes autopoiétiques sont intrinsèquement auto-référentiels, leurs états dynamiques n'étant déterminés par rien d'extérieur ${ }^{37}$. Cette clôture opérationnelle implique ce que Jean-Pierre Dupuy nomme " endocausalité », à savoir une forme de causalité intérieure qui définit précisément les systèmes autopoïétiques : «l'être autonome n'a d'autre cause et d'autre effet que lui-même. Il n'est le produit d'aucun projet, d'aucun programme, ce programme serait-il extérieur à l'être autonome ou bien intérieur, inscrit dans l'une de ses parties. Il est à lui-même, globalement, son propre programme $»^{38}$. Cette conception permet à Strauss de réactualiser l'idée ancienne selon laquelle chaque livre est fait de tous les livres et que tous les livres ne sont que les fragments d'un seul livre infini. Un livre qui s'écrit lui-même, selon les lois d'une "génétique littéraire ", qu'il modèle sur les lois du vivant. Or dans le vivant, ce qui caractérise la transmission du matériel génétique, c'est qu'elle ne se fait jamais sans perte. L'information génétique est copiée, redupliquée en vue de sa transmission, mais celle-ci ne s'opère jamais sans perte, sans « erreurs de copie ».

Le biologiste Henri Atlan a proposé de considérer ces «erreurs de copie» comme des facteurs positifs permettant aux systèmes biologiques de se réorganiser à un niveau de complexité supérieur, dans un autre contexte, émergent ${ }^{39}$. En introduisant l'idée de niveaux d'organisation, il a montré que l'information perdue ou détruite au niveau inférieur pouvait représenter, au niveau d'intégration supérieur, un accroissement d'information. Comme dans toute hiérarchie organisée, le passage d'un niveau élémentaire à un niveau plus intégré comporte en effet la possibilité de ressaisir le désordre en complexité. Pensée sur ce modèle, la littérature apparait comme un système doté des mêmes capacités d'auto-organisation que les organismes naturels : « les erreurs de copie » (d'interprétation) au niveau du texte peuvent avoir un effet positif au niveau du système littéraire, car elles rendent possible le renouvellement de la parole vivante, du savoir et de l'écriture. Livrée aux aléas de la mémoire et à l'errance de l'histoire, la littérature se génère en effet à la faveur de malentendus, d'erreurs d'appréciation ou d'interprétation, de transformations créatrices et de pertes, qui permettent au matériel écrit de s'enrichir et de se diversifier. Loin d'entraver l'écriture ou de paralyser la création, ces écarts permettent d'accroitre la diversité et donc la complexité par suppression des redondances. Dans Les erreurs du copiste ${ }^{40}$, un ouvrage de 1997, Botho 
Strauss a décliné cette idée d'après le modèle biologique de l'auto-organisation, qui permet d'appréhender le désordre comme source de créativité.

\section{Émergence et auto-organisation} instables à adopter une structure nouvelle, qui correspond à un accroissement d'ordre et de complexité. Cette tendance est notamment celle des organismes vivants qui sont éloignés de l'équilibre, ce qui peut les amener, une fois passé un seuil critique de complexité, à s'organiser eux-mêmes, sans l'intervention d'une source extérieure ${ }^{41}$. Typiquement, les systèmes auto-organisés possèdent des propriétés émergentes, c'est-àdire telles qu'elles n'auraient pas pu être prédites à partir de la seule connaissance du niveau auquel elles apparaissent. Complémentaire de la notion d'auto-organisation, la notion d'émergence permet de "penser techniquement l'imprévisible à défaut de connaître totalement l'irréversible $»^{42}$. Elle sert surtout à décrire les systèmes à hiérarchie enchevêtrée, qui ont au moins deux niveaux différents d'organisation observables à deux échelles spatio-temporelles différentes. A cette première condition s'ajoute l'existence, au niveau sous-jacent, de phénomènes collectifs et coopératifs, distribués et complexes, dont la dynamique peut faire émerger, au niveau macroscopique, des états organisationnels globalement cohérents et morphologiquement structurés. Des états qui restent pourtant indépendants de la structure fine du niveau inférieur, manifestant ainsi une autonomie de l'organisation globale par rapport aux interactions locales dont elle émerge.

Les théories de l'auto-organisation ont contribué à renouveler notre compréhension des concepts biologiques de croissance, de différenciation et d'évolution, puisque le nouveau peut désormais être pensé comme une conséquence directe de l'instabilité des systèmes, en dehors des schémas progressifs et linéaires. Dans un écosystème par exemple, la stabilité est assurée par l'interaction des diverses espèces qui le composent et qui lui permettent de résister aux sollicitations du milieu extérieur. Mais lorsque celles-ci sont trop fortes, le système ne peut plus s'adapter, il voit surgir une « catastrophe » qui signe la fin de sa capacité d'adaptation. Il y a alors transition vers un autre état: c'est l'autoorganisation, processus par lequel le système innove en luttant contre les conditions extérieures, se réorganise de l'intérieur et produit un nouvel ordre plus complexe. Dans ce modèle, la complexité n'est plus le seul résultat de la sélection naturelle mais un produit de la dynamique interne des systèmes, qui sont amenés à adopter des formes toujours plus complexes et plus différenciées que modèle la sélection. On peut ainsi comprendre le passage des organismes monocellulaires aux organismes pluricellulaires à l'ère cambrienne. Alors que le vivant avait jusque-là perpétué à l'infini la même uniformité, soudain et de façon spectaculaire, la différenciation cellulaire fit son apparition, entraînant une extraordinaire explosion de formes nouvelles, différenciées, dont les degrés de complexité étaient infiniment variables: de l'homogène émergeait l'hétérogène, de la simplicité la complexité, de la catastrophe un ordre nouveau.

Les théories de l'auto-organisation ont aussi contribué à renouveler l'approche des problèmes de morphogenèse, qui sont aujourd'hui abordés sous un angle à la fois plus dynamique et plus ouvert à l'aléa. Désormais, les sciences du vivant insistent en effet sur l'instabilité comme régime de fonctionnement des systèmes naturels, elles reconnaissent que leurs objets « ne sont pas seulement des systèmes capables de conserver/reproduire des structures stables dans des conditions instables et de réguler/programmer leurs 
opérations. Ce sont aussi des êtres qui déploient une variété de formes et une créativité morphologique dont les naturalistes de tout temps se sont émerveillés ${ }^{43}$. De nouvelles conceptions morphodynamiques sont ainsi apparues, qui appréhendent la forme, non plus comme structure stable, mais comme auto-organisation émergente pouvant être décrite à partir des dynamiques locales qui sont à son origine. La morphodynamique de Jean Petitot s'inscrit dans ce cadre épistémologique, élargi afin de pouvoir rendre compte des structures formelles du niveau symbolique, c'est-à-dire des formes esthétiques ${ }^{44}$. Son ambition est de réconcilier l'objectivité physique avec le monde de la forme et du sens : non pas le sens des herméneutes comme le souligne Petitot, mais celui du monde sensible, des formes, des choses et des processus ${ }^{45}$. Le sémiologue s'inscrit ainsi dans la lignée de Goethe, qui est le premier à avoir esquissé une conception de la forme comme réalité émergente irréductible au support sur lequel elle survient pourtant. Petitot s'est aussi intéressé de près à la pensée morphogénétique de Valéry, qu'il décrit comme la conjugaison entre un pouvoir d'auto-organisation et une spontanéité qui défie la loi de l'entropie, appelant ainsi à une conception génétique de la forme comme "autoorganisation émergente $»^{46}$. Le fil rouge qui relie Goethe à Valéry et Petitot est une approche à la fois dynamique et phénoménale de la forme qui, loin de se constituer contre le sensible, part d'une " phénoménologie de l'apparent » : prenant la nature dans son immédiateté, telle qu'elle se donne dans le monde de la perception ordinaire, elle cherche pourtant à en donner une description physique objective, fondée sur l'observation de ses processus de formation et de trans-formation.

Avec les concepts d'émergence, d'auto-organisation ou d'autopoï̀se, la biologie contemporaine offre de nouvelles ressources conceptuelles et expressives appropriables par l'art. De cette disponibilité témoignent le développement depuis une quarantaine d'années du «bio-art ", les expériences de simulation de la vie sur ordinateur ou encore l'affirmation de champs critiques concernés par les rapports entre le vivant et la littérature (animal studies, écocritique, biopoétique, etc.). Pour n'en donner qu'un exemple, on peut évoquer le travail de l'artiste-ingénieur Karl Sims qui, en 1987, s'est efforcé de synthétiser dans ses Locomotion Studies des créatures capables de reproduire les fonctions essentielles des êtres vivants. A partir d'un génotype programmé, dont il faisait évoluer à la fois l'architecture de contrôle et la morphologie, il a produit des hybrides d'art et de technique, d'informatique et de biologie ${ }^{47}$. En 1994, il a créé, grâce à de puissants ordinateurs, des créatures virtuelles baptisées Evolvable virtual creatures, sortes d'agrégats morphologiques qui répondent à une élaboration heuristique du principe darwinien de la sélection et des théories de l'auto-organisation. Conçues pour déterminer leur capacité de survie par régulation et adaptation, elles peuvent adopter les comportements les plus variés : évoluer, muter, s'adapter en adoptant des stratégies similaires à celles observées dans l'évolution biologique. On peut mesurer la portée épistémologique et éthique de cette expérience lorsqu'on sait que certaines de ces entités virtuelles sont conçues pour « retracer l'hypothèse darwinienne du passage, en un temps immémorial, de l'eau à la terre ferme pour l'être vivant... $»^{48}$. Esthétique et épistémologie se conjuguent ici dans une même quête d'intelligibilité qui, faisant fi des frontières entre arts et sciences, les fait converger dans une œuvre d'art qui est en même temps un dispositif cognitif. 


\section{Esthétique, biologie et cognition}

24 la perception », la forme n'existant qu'à travers des processus cognitifs qui lui permettent de s'actualiser. Les Grecs le savaient bien, qui n'établissaient pas d'opposition radicale entre ce qui voit et ce qui peut être vu. Les trois termes dont ils disposaient pour désigner la forme - eidos, idéa, morphê - renvoyaient à la fois à ce qui se présente à la vue et au phénomène de la vision: autrement dit, à la configuration reconnue (par les yeux du corps ou de l'esprit) et à son origine. Ce qui est une manière de rappeler qu'il n'y a de forme qu'en rapport avec les formes de la sensibilité, avec les moules et grilles de la perception et de l'entendement humains. Goethe, dans les textes autobiographiques entourant $\mathrm{La}$ Métamorphose des plantes, a repris cette idée d'une morphogenèse se développant conjointement au sein de l'objet et du sujet, pour tisser un lien entre la forme naturelle vivante, la forme artistique et la forme du sujet. Dans une lettre du 6 juin 1798 adressée à Schiller, il soulignait la nécessité de comprendre la formation et la transformation des formes en tant qu'elles apparaissent à l'esprit humain :

J'accorde très volontiers que ce que nous connaissons n'est pas la nature elle-même, et qu'elle n'est reçue en nous que selon de certaines formes et de certaines capacités de notre esprit. Entre l'envie qu'a l'enfant de cueillir la pomme qui pend à l'arbre et la chute de cette pomme, qui passe pour avoir suggéré à Newton la première idée de sa théorie, il se peut fort bien qu'il y ait toute une échelle de degrés dans l'intuition dont on ferait bien de nous dresser une bonne fois, bien au complet, le tableau, en nous donnant clairement à entendre ce que l'on considère comme le degré suprême. ${ }^{49}$

Réhabilitant la forme sensible, Goethe ne sépare pas la forme phénoménale de l'œil qui rend son appréhension possible, suggérant ainsi une solidarité entre l'organisation biologique, la structuration de la perception et l'esthétique. S'il sollicite cette dernière, ce n'est pas seulement au sens d'une science générale de la sensibilité mais aussi d'une étude sur notre réception face aux «belles formes " produites par l'art ${ }^{50}$. Son objectif est en effet de saisir le "surplus de psychologie des formes" qui se greffe aux choses dès qu'elles sont perçues par l'homme. Il faudra cependant attendre le $\mathrm{xx}^{\mathrm{e}}$ siècle pour que ce «surplus de psychologie» soit formalisé par Wolfgang Koehler, le fondateur de la Gestaltpsychologie. La Gestaltpsychologie est une approche phénoménologique de la forme dont l'approche synthétique et englobante est en rupture avec le paradigme analytique et mécaniste des sciences positives. Dans l'esprit de ses créateurs, elle devait être une théorie universelle des formes et des organisations, ayant vocation à englober dans un même cadre théorique les processus physiques, perceptifs et esthétiques. Utilisable dans une pluralité de champs de connaissance, la notion de Gestalt, que Köhler emprunte à Goethe, devait permettre d'unifier toutes les disciplines appelées à affronter le problème des formes et de l'organisation, contribuant ainsi à une nouvelle articulation de la nature et de la culture ${ }^{51}$. C'est là toute l'originalité de la perspective gestaltiste : elle a conçu une théorie générale des formes qui implique la nécessité de sa détermination pluridisciplinaire (psychologie, biologie, physique) en même temps que la possibilité d'une traduction illimitée d'un discours à l'autre ${ }^{52}$. Köhler mobilisera donc les méthodes techniques et instrumentales des sciences de la nature mais il refusera la réduction physicaliste pure et simple, faisant partir l'enquête psychologique du monde de l'expérience immédiate, tel qu'il s'offre sans détour et spontanément dans la perception. 
Le monde des Gestalten est en effet le monde des apparences et de l'apparaître, l'expérience qualitative de notre rapport premier au monde, le monde phénoménal. somme des parties qui lui préexistent; il y a au contraire, et immédiatement, une structure globale du champ qui se déploie dans chacune de ses parties; simultanément, celles-ci agissent les unes sur les autres et se déterminent mutuellement ${ }^{55}$. C'est donc toujours le global qui détermine le local, il y a détermination réciproque et dynamique de la structure et du procès. Or c'est précisément dans cette idée d'une totalité organique transcendant la somme de ses composantes, que la Gestalpsychologie retrouve l'esthétique. Elle la retrouve également dans l'idée d'une dynamique propre aux Gestalten, appelée «tendance à la prégnance» (Prägnanz). Définie comme une «orientation souvent reconnue dans l'esthétique $\aleph^{56}$, qui consiste pour la nature à adopter la forme la plus simple et la plus distincte possible, la notion de prégnance s'ouvre sur l'idée moderne de structure, "comprise comme le répondant objectif d'un processus de construction qui reste profondément sous-déteminé tant qu'une norme culturelle n'est pas venue le fixer " ${ }^{57}$. Ce qui a pour effet de changer le statut de la «bonne forme»: "elle n'est plus seulement l'effet d'une couche esthétique superficielle, mais le produit sensible de principes d'organisation inhérents à toute perception $\aleph^{58}$. C'est dans le domaine de la perception visuelle que la notion, corrélative, de saillance et la distinction entre figure et fond ont été explorées, avec des approches et des objectifs variés. La notion de saillance est liée à l'émergence d'une figure sur un fond, que cette émergence soit motivée par des aspects physiques liés à la perception ou par des aspects plus sémantiques, voire cognitifs. Ces deux notions ont été redéfinies par René Thom ${ }^{59}$, qui est parvenu à donner un modèle fonctionnel de reconnaissance des formes dans la perception visuelle, en envisageant l'organisation fondamentale de notre cognition comme une interaction dynamique de saillances et de prégnances dans un espace géométrique. En associant en un tout cohérent la physiologie de la vision et les mécanismes mentaux, il a donné un prolongement au projet gestaltiste d'une théorie unitaire des formes biologiques, des formes esthétiques et des structures cognitives.

Aujourd'hui, ces thèses ont fait place à des modèles qui réintègrent la cognition dans le corps vivant tout en la situant dans un environnement écologique : le cerveau existe dans un corps, le corps existe dans le monde, la connaissance est donc incarnée (embodied cognition) et on peut alors parler "d'énaction", selon le néologisme introduit par Francisco Varela. Ce dernier considère le système nerveux comme un système dont le fonctionnement ne peut être compris qu'en rapport avec le corps dans lequel il existe : un corps qui, pour se développer, a besoin de saisir l'information venue des sens. Or cette information ne lui parvient pas sous une forme brute, directement de la réalité 
extérieure, mais traduite dans le seul langage accessible au cerveau : le langage unifié des potentiels nerveux. Autrement dit, nos perceptions ne naissent pas dans les organes sensoriels mais dans le cerveau qui façonne le monde selon des lois et des règles qui lui sont propres. Ainsi, chaque organisme crée son domaine cognitif de manière à maintenir son identité organisationnelle. Une conséquence de cette clôture opérationnelle est que la réalité n'apparaît plus comme préexistante à l'acte cognitif: elle n'existe pas préalablement (comme dans les logiques de la représentation), elle n'est pas non plus construite in subjecto, mais elle surgit d'interactions constantes entre le cerveau et un corps qui vit et agit dans un environnement déterminé. Construisant des mondes en même temps qu'il construit sa propre identité, le cerveau fonctionne sur le principe d'une inséparabilité entre identité et connaissance : l'une n'est pas préalable à l'autre; elles émergent en même temps : chaque fois qu'un objet constitue son identité, cela crée, automatiquement, un monde cognitif et une situation de connaissance ${ }^{60}$. L'image de la cognition qui en découle est un «faire-émerger créateur d'un monde, avec la seule condition d'être opérationnel : elle doit assurer la pérennité du système en jeu ${ }^{61}$.

Dans L'incommencement, Botho Strauss a tiré toutes les conséquences de ce que le psychologue Ernst von Glasersfeld a qualifié de « constructivisme radical » ${ }^{62}$. En faisant du cerveau un organe qui construit des mondes plutôt que les réfléchir, Varela efface en effet les frontières entre poésie et cognition : «Le cerveau traite le monde extérieur en le créant. La poésie réagit par un désir analogue d'être autonome. Jamais elle ne doit quoi que ce soit à une impression immédiate. ${ }^{63}$ Se déployant « en dehors des poèmes et des œuvres de prose", la poésie peut dès lors être envisagée comme une activité fondamentale, inscrite au cœur même de notre être biologique, et non plus comme une activité seconde, vouée à reproduire un monde d'abord façonné par nos sens :

Comme il nous faut sans cesse traduire toute chose dans notre mode, sous peine de ne comprendre aucun monde, comme nous ne pouvons survivre que comme d'incessants producteurs d'images du monde, il n'est guère étonnant que création et fabrication, poésie et poïesis, en tant que continuation et mesure de l'activité cognitive, soient à la nature humaine ce que le vol est aux oiseaux. Voilà pourquoi le poète prétendait autrefois à la prééminence, étant le plus efficace parmi les transformateurs que nous sommes, nous qui, depuis le premier battement de notre cœur, sommes machines à inventer et depuis notre première pensée devons venir à bout de quelque chose d'inconcevable. ${ }^{64}$

Libérée de la réalité extérieure, affranchie des limites de la matière ou du but, l'activité créatrice ne se heurte plus à aucune résistance, elle acquiert une légitimité absolue, qui témoigne du double enracinement des formes artistiques : produits de l'activité d'un être vivant, elles sont artificielles, extérieures à sa propre forme, mais pourtant elles le prolongent, lui permettent de s'enrichir et de s'épanouir. Car l'art fait aussi partie de la vie : il en est une production au même titre que les formes naturelles.

31 La création apparaît ainsi comme la loi même de l'Être, une loi qui s'enracine " d'une part dans la structuration morphologique du monde naturel et de l'autre, dans le corps propre, la perception et l'action $»^{65}$. D'où la nécessité d'une approche capable d'unifier ce que l'histoire a séparé : la biologie, la cognition et l'esthétique. Fondée sur l'analogie entre l'art et le vivant, une telle approche est à la fois source de création et méthode de connaissance comme en témoignent les exemples discutés ici, qui rendent au mot " esthétique » sa double valence de «science des arts autant qu'art des sciences » ${ }^{66}$. 


\section{NOTES}

1. Paul Valéry, "Le Cimetière marin ", Paris, Gallimard, 2016. Le poème a d'abord été publié en 1920 en revue avant d'être repris dans Charmes (Éditions de la Nouvelle Revue française, 1922). Le vers est tiré de la strophe suivante « $O$ pour moi seul, à moi seul, en moi-même, / auprès d'un cœur, aux sources du poème, / entre le vide et l'événement pur, / j'attends l'écho de ma grandeur interne, / amère, sombre et sonore citerne, / sonnant dans l'âme un creux toujours futur!», p. 187-188.

2. Horst Bredekamp, Les coraux de Darwin, Paris, Les presses du réel, 2008, p. 47. Le seul dessin à illustrer De l'origine des espèces est un schéma stylisé et abstrait de l'évolution des espèces, qui figure la parenté de tous les êtres vivants, ceux qui nous entourent et ceux qui ont depuis longtemps disparu. Ce dessin, le fameux arbre de vie, a répandu une image de l'évolution comme arbre schématisé, conformément à la doxa darwinienne. Or cette interprétation repose sur un malentendu où les bras des coraux ont été pris pour des branches d'arbres. Le branchage, dans les diagrammes de Darwin, ne renvoie pas à la métaphore des branches d'un arbre, mais à celle des bras d'un corail, le système pétrifié des branches représentant les restes fossilisés.

3. Ibid., p. 135.

4. "The tree of life should perhaps be called the coral of life ". Darwin, Darwin's Notebooks 1836-1844, geology, transmutation of species, metaphysical enquiries, Paul H. Barrett et Peter J. Gautrey eds., Londres, British Museum (Natural History), 1987, p. 177.

5. Ibid.

6. Voir sur la question l'ouvrage déjà cité de Horst Bredekamp, Les coraux de Darwin, op. cit., p. 103.

7. "We feel surprised, when travellers relate accounts of the vast extent of certain ancient ruins; but how utterly insignificant are the greatest of these, when compared to the pile of stone here accumulated by the work of various minute animals", Charles Darwin, Voyage of the Beagle: Charles Darwin's Journal of researches, Janet Browne and Michael Neve eds, Londres, Penguin Books, 1989, p. 338. Paru en français sous le titre Voyage d'un naturaliste autour du monde : fait à bord du navire le «Beagle » de 1831 à 1836, tr. fr. Edmond Barbier, Paris, La Découverte, 2003 (1875), p. 490.

8. Selon l'expression de Horst Bredekamp, op. cit.

9. Ovide, Les Métamorphoses, traduction française Danièle Robert, Arles, Actes Sud, 2001, Livre IV, Vers 750-752, p. 189.

10. Darwin a développé ce principe dans The Descent of man and selection in relation to sex [La descendance de l'homme et la sélection sexuelle], Richard Dawkins ed., Londres, Gibson Square Books, 2003 (1871), p. 423 sq. Voir sur la question le livre de Horst Bredekamp, op. cit., p. 130-131.

11. Pour le domaine littéraire, on peut citer pour exemple l'étude de George Levine, " "And if it be a pretty woman, all the better" - Darwin and sexual selection ", Helen Small \& Trudi Tate eds, Literature, Science, Psychoanalysis 1830-1970, Essays in Honour of Gillian Beer, Oxford, Oxford University Press, 2003, p. 44 sq. Pour l'esthétique, mentionnons l'étude de Winfried Menninghaus, Das Versprechen der Schönheit, Frankfurt am Main, Suhrkamp, 2003, p. 67-157. Enfin, pour l'histoire de l'art, nous renvoyons au travail de Julia Voss, «Augenflecken und Argusaugen. Zur Bildlichkeit der Evolutionstheorie", in Bildwelten des Wissens. Kunsthistorisches Jahrbuch für Bildkritik 1, 2, 2003, p. 75-85.

12. Parmi eux, on peut citer les noms de Schiller, Friedrich Schlegel, Eckermann, Hegel... Dans le monde allemand, la pensée morphologique goethéenne a été diffusée grâce aux efforts du fondateur de l'anthroposophie, Rudolf Steiner (1861-1925), qui voyait en lui le père d'une nouvelle esthétique et le fondateur d'une nouvelle épistémologie, qu'il a contribué à faire 
essaimer dans des domaines aussi variés que les arts plastiques, l'architecture, la littérature, la philosophie et finalement la science elle-même. L'esprit scientifique goethéen, s'il n'a pas participé aux grandes révolutions $\mathrm{du} \mathrm{xx}^{\mathrm{e}}$ siècle, a donné naissance à une autre rationalité savante dont les principes continuent à nourrir quelques esprits hétérodoxes. Comme par exemple Theodor Schwenk, hydrodynamicien et théosophe, inventeur d'une technique pour mesurer la pureté de l'eau mais surtout connu comme auteur d'un ouvrage intitulé Le chaos sensible, salué à sa sortie en 1962 (1982 pour la traduction française), comme la première monographie phénoménologique de l'eau et de l'air. Plus près de nous, on peut évoquer la pensée de René Thom telle qu'est exposée par exemple dans Paraboles et catastrophes. Entretiens sur les mathématiques, la science et la philosophie, Paris, Flammarion, 1983, p. 133 sq. Pour sa part, Jean Petitot (Morphologie et esthétique, Paris, Maisonneuve et Larose, 2003, p. 72-74) retrace une généalogie qui part de Goethe pour aboutir au structuralisme en passant par Vladimir Propp, Claude Lévi-Strauss ou encore l'historien de l'art néerlandais André Jolles (1874-1946).

13. Goethe, Betrachtung über Morphologie [Observation sur la morphologie], HA XIII [Hamburger Ausgable], 1949, vol. XIII, p. 124. Cette édition, dite de Hambourg, a paru entre 1949 et 1981, son directeur de publication est Erich Trunz (München, C. H. Beck Verlag). Le volume XII contient les Maximes et réflexions, les volumes XIII et XIV contiennent l'essentiel des grands écrits scientifiques (botanique, zoologie, géologie, anatomie, chromatologie).

14. Goethe, Die Absicht eingeleitet [L'intention introduite], HA XIII, Ibid., p.55-56. Cité aussi dans René Michéa, Les travaux scientifiques de Goethe, Paris, Aubier-Montaigne, 1943, p. 170-171.

15. Jean Petitot, Morphologie et esthétique, op. cit., p. 14.

16. Goethe, Maximen und Reflexionen, HA XII, n 720, p. 467, Trad. Deshusses modifiée, p. 81.

17. Danièle Cohn, La lyre d'Orphée. Goethe et l'esthétique, Paris, Gallimard, 1999.

18. Ce n'est pas un hasard si Goethe recourt souvent à l'expression « regarder les objets avec les

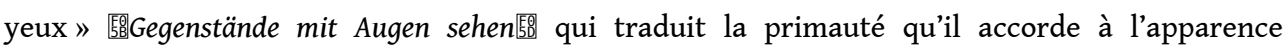
phénoménale. Voir son Journal de voyage, Venise 10 octobre 1786. In HA XI. Cité par Jean-Jacques Wunenburger, in Goethe et la Naturphilosophie, Mai Lequan ed., Paris, Klincksieck, 2011, p. 63-74, p. 67.

19. Patrice Maniglier, «Du mode d'existence des objets littéraires: enjeux philosophiques du formalisme ", Les temps modernes n 676, nov-déc. 2013, p. 48-80, p. 73.

20. J. W. Goethe, Essai sur la métamorphose des plantes, traduction française Frédéric Soret, Genève, éditions Notari, 2010, § 50, p. 79.

21. Jean-Jacques Wunenburger, « Goethe, notes sur une épistémologie alternative », op. cit., p. 67.

22. Goethe, Italienische Reise, « Palermo, Dienstag den 17. April 1787 », HA XI, op. cit., p. 266-267, trad. Mutterer modifiée, Paris, Champion, 1931, p. 267-268.

23. Didier Hurson, «Goethe et la question du déterminisme. Les concepts goethéens en biologie et en physiologie : épigenèse et préformisme, déhiscence et métamorphose ", in Goethe et la Naturphilosophie, op. cit., p. 111-129.

24. Ibid., p. 119

25. Laurent van Eynde, «En quoi la Naturphilosophie est-elle proprement philosophique?», in Goethe et la Naturphilosophie, op. cit., p. 15-32, p. 24.

26. Patrice Maniglier, «Du mode d'existence des objets littéraires: enjeux philosophiques du formalisme », op. cit., p. 74.

27. Maurice Merleau-Ponty, Notes de cours au Collège de France, 1952-1961, mss., vol. VIII, p. 15.

28. Paul Valéry, Cahiers 2, édition établie, présentée et annotée par Judith Robinson-Valéry, Paris, Gallimard, Bibliothèque de la Pléiade, 1974 (1921-1922), p. 342.

29. Paul Valéry, «L'homme et la coquille » (1937), Euvres 1, édition établie et annotée par Jean Hytier, Paris, Gallimard, Bibliothèque de la Pléiade, 1957 (1957), p. 897.

30. Ibid., p. 900.

31. Ibid., p. 896. 
32. Francisco Varela et Humberto Maturana, L'arbre de la connaissance, Paris, Addison-Wesley, 1994, p. 32. Titre original: The Tree of Knowledge: The Biological Roots of Human Understanding, Boston, New Science Library, 1987.

33. Valéry, "L'homme et la coquille", op. cit., p. 900.

34. Valéry, Cahiers 1, édition établie, présentée et annotée par Judith Robinson-Valéry, Paris, Gallimard, Bibliothèque de la Pléiade, 1973, p. 562.

35. Ibid., p. 1342.

36. Botho Strauss, « Die Erde, ein Kopf. Dankrede zum Büchnerpreis 1989 », in Die Zeit 27.10.1989, p. 65-66. Repris dans Der Aufstand gegen die sekundäre Welt. Bemerkungen zu einer Ästhetik der Anwesenheit. München/Wien 1999 (1990), p. 23-35. Ici p. 29. La traduction est faite par mes soins.

37. Pour autant, les systèmes aupoïétiques ne sont pas coupés de tout échange d'énergie avec leur environnement, bien au contraire: la clôture en effet ne concerne pas les interactions avec l'environnement mais elle signifie seulement que tout contact avec l'extérieur est assujetti aux conditions du système et non à celles de l'environnement. Voir Louis-José Lestocart, Entendre l'esthétique dans ses complexités, Paris, L'Harmattan, 2008, p. 134.

38. Cité par Louis-José Lestocart, ibid., p. 132.

39. Voir ses travaux sur la question : Le cristal et la fumée. Paris 1979. Voir aussi «L'émergence du nouveau et du sens ", in L'auto-organisation, J.-P. Dupuy et P. Dumouchel dirs., Paris 1983, p. 115-130. Voir enfin "Créativité biologique et auto-création du sens ", Cahiers du CREA 9, 1986, p. $145-191$.

40. Botho Strauss, Les erreurs du copiste. Traduction française de Colette Kowalski, Paris, Gallimard, 2001 (1997).

41. Jean Petitot, "La sémiophysique: de la physique qualitative aux sciences cognitives", in Passion des formes. A René Thom, Michèle Porte dir., Paris, ENS éditions, 1994, p. 499-546, p. 502.

42. Jean-Claude Beaune, "Lueurs sur le temps des techniques", in Les figures du temps, JeanJacques Wunenburger et Lambros Couloubaritsis dirs, Presses Universitaires de Strasbourg, 1997, p. 300.

43. Anne Fagot-Largeault, «Le vivant ", in Notions de Philosophie I, Denis Kambouchner dir, Paris, Gallimard, coll. Folio/essais, 1995, p. 289.

44. Petitot se réclame des théories de René Thom qui, dès les années 1970, se proposait de développer « une méthode universelle, permettant d'associer à toute apparence morphologique une situation dynamique locale qui l'engendre, et cela de manière indépendante du substrat matériel ou non, vivant ou non vivant - qui en est le support ». Cité par Michèle Porte, in Passion des formes, op. cit., p. 29.

45. Jean Petitot, in Passion des formes, op. cit., p. 502.

46. Jean Petitot, Morphologie et esthétique, op. cit., p. 120.

47. Louis-José Lestocart, Entendre l'esthétique dans ses complexités, op. cit. p. 125.

48. Ibid., p. 83.

49. Goethe-Schiller. Correspondance 1794-1805, Traduction et avant-propos de Lucien Herr. Nouvelle édition revue, augmentée et présentée par Claude Roëls, Paris, Gallimard, 1994, p. 16.

50. Nicolas Class, « De l'usage du canon esthétique dans les sciences naturelles chez Goethe et de ses présupposés philosophiques ", in Goethe et la Naturphilosophie, op. cit., p. 33-62, p. 37.

51. Yves-Marie Visetti et Victor Rosenthal, Köhler, Paris, Les Belles lettres, 2003, p. 13.

52. Ibid., p. 47.

53. Wolfgang Koehler, «Bemerkungen zur Gestalttheorie. Im Anschluss an Rignanos Kritik », Psychologische Forschung 11, 1928, p. 188-234, p. 224.

54. Yves-Marie Visetti et Victor Rosenthal, Köhler, op. cit., p. 51-53.

55. Ibid., p. 65.

56. Wolfgang Koehler, The Task of Psychology, Princeton, Princeton University Press, 1969, p. 58.

57. Yves-Marie Visetti et Victor Rosenthal, Köhler, op. cit., p. 230. 
58. Ibid. Pour ne citer que quelques-uns des auteurs à avoir donné des prolongements aux thèses gestaltistes dans le domaine esthétique, on peut évoquer les noms de R. Arnheim, E. Gombrich ou E. Panofsyky. Dans le domaine de la littérature, le cas de Robert Musil est exemplaire puisqu'il a étudié à Berlin avec les pères fondateurs de l'école gestaltiste et qu'il a tenté de transposer leurs idées à la compréhension du système littéraire.

59. René Thom, Esquisse d'une sémiophysique. Physique arstotélicienne et théorie des catastrophes, Paris, InterEditions, 1988. La sémiophysique peut être envisagée comme une ontologie des prégnances et des saillances, où la signification procède de passages singuliers entre le formel et le sémantique, les formes et les dynamiques, l'expression et l'intention, et réciproquement. Thom a joué un rôle fondamental dans les années 1960-1970 en proposant une théorie de la forme qui concilie gestaltisme, structuralisme et genèse formelle grâce à un usage à la fois rigoureux et très général du concept mathématique d'instabilité. Dans cette optique, toute morphologie se comprend comme le résultat d'un processus de stabilisation par lequel se réalise la différenciation progressive d'un espace donné. Autrement dit, l'instabilité est considérée comme un germe initial à partir duquel toute ontogenèse, toute individuation peut être comprise comme une stabilisation. Voir sur ces questions ses ouvrages : Stabilité structurelle et morphogenèse, Paris, InterEditions, 1972 ; et Modèles mathématiques de la morphogenèse, Paris, Union générale d'édition 10/18, 1974.

60. Voir l'entretien accordé par Varela à la revue TLE, dans TLE $n^{\circ} 8$, Publication de l'Université de Vincennes, 1990, p. 171.

61. Francisco Varela, Connaître les sciences cognitives, Tendances et perspectives, Paris, Seuil, 1989, p. 112.

62. Ernst von Glasersfeld, "The radical constructivist view of science ». in A. Riegler ed., Foundations of Science, special issue on "The Impact of Radical Constructivism on Science", vol. 6, $\mathrm{n}$ - 1-3, p. 31-43, 2001.

63. Botho Strauss, L'Incommencement. Réflexion sur la tache et la ligne, traduction française de Colette Kowalski, Paris 1996 (1992), p. 68.

64. Ibid., p. 29.

65. Jean Petitot, Morphologie et esthétique, op. cit., p. 136

66. Jean-Louis Lemoigne, "Le "Disegno" est d'une excellence telle... ", postface au livre de LouisJosé Lestocart, Entendre l'esthétique dans ses complexités, Paris, L'Harmattan, 2008, p. 188.

\section{RÉSUMÉS}

Cet article explore les liens qui, depuis l'Antiquité, lient les savoirs du vivant à l'esthétique, entendue à la fois comme science du beau, comme science des formes et comme théorie de la réception des œuvres d'art. Sans prétendre à l'exhaustivité ou à la continuité historique, il braque le projecteur sur des penseurs - scientifiques, poètes ou artistes - qui ont marqué l'histoire des relations entre les deux domaines: Goethe, Darwin, Köhler, Valéry, René Thom, Botho Strauss...

\section{INDEX}

Mots-clés : vivant, esthétique, histoire de l'art, cognition, forme 


\section{AUTEUR}

\section{LAURENCE DAHAN-GAIDA}

Université de Franche-Comté, Réseau VIVANLIT 